\title{
Entropic potential as manifold for the reduced entropy representations in superconductivity
}

\author{
Z. Bousnane ${ }^{1}$, M. Benslama ${ }^{2}$, N. Merabtine ${ }^{2}$, \\ ${ }^{1}$ Physics Department, Faculty of Science, University of Batna, 05000 Algeria \\ ${ }^{2}$ Electromagnetism and Telecommunication Laboratory, Electronics Department, \\ Faculty of Engineering, University of Constantine, 25000 Algeria \\ Corresponding author: malekbenslama@hotmail.com
}

\begin{abstract}
The "cold" causes superconductivity phenomenon, as a measurement process generating a phase transitions of the second order, and also permitting the rise of phenomenological parameters, but not allowing the stability of such behaviour, we will have to write this stability to be supported by a new kind of value as an entropic potential which learns the systems how to display over all possible microconfigurations, and the systems by their faculties to increase their standard entropies, dictate the form of this potential, this will be written as a recursive operatorial equations. The picture of such potential is interference between thermodynamical length scales of entropies, it seems as a world of different undetermination orders, if a given variational principle is put down, it will transit over several definitions. The representation of the entropic potential as a group transformation linking different temperature thermodynamical length scales as described by L. Landau will suggest that among considerations brought on propagation of order parameter carving the recursive stationary state. Concerning the stability conditions, a context of matrices description will be proposed, as managing the fact that superconductor, is superconductor per pavement, and the matrixes notations, is determining the interaction between order parameters as "Heredity" concerning dimensions, orientations and a mixed subinteraction, where equivalently dimensions and orientations are mutually excluded each other. The beginning of emergence of this value is rooted when combinatory formulation of matter, is taken in the order of measurement process with eigen results, corresponding to normalised macroscopicity levels. The asymptotic expression for the entropic potential, will be expressing a physical situation defining that, deflections are collected by a variational principle brought on the Landau length coherence. The introduction of covariant, contravariant and mixed current density matrices will establish the limit, according which the reproducible recursive configurations is only obtained by triplication of a combined fundamental states.
\end{abstract}

Keywords: entropic potential, entropy, thermodynamical length scales, superconductivity.

Manuscript received 30.11.06; accepted for publication 26.03.07; published online 01.06.07.

\section{Introduction}

Mathematical modelling and simulation have become an essential part of superconductivity engineering analysis [1], The study of Ginzburg-Landau model permit us the best understanding of eigen energy system [2]. It had been demonstrated that the vortex is the leading aim of the particles motion [3]. Besides, an alternative approach based on Bardeen-CooperpSchrieffer (BCS) theory explores the effects of local pairing correlations and leads to the concept of
"Cooperon propagator" [4]. Focusing our attention on scaling theory, we can explore bicritical phenomenon as mentioned by [5], and weak coupling as pointed by [6].

We will have to introduce an entropic potential operator (which will show the global macro behaviour displaying in regards to the states described by the order parameter), by considering that the Landau potential distribution, will be a result of measurement process, as interaction between number of states described by order parameter and entropic potential, the number of states must obey to a phenomenological critical law. 


\section{The Landau thermodynamical length scales for entropic potential}

By introducing reduced entropy $S_{\alpha \beta}$ as a matrix of the form:

$\mathrm{S}_{\alpha \beta}=-5.0396 \cdot 10^{-15}\left(\ln _{\beta} \Delta \Gamma\right)$

as $\ln \Delta \Gamma_{\alpha} \Delta \Gamma_{\beta}$, where $\Delta \Gamma$ is the number of states described by the order parameter, which will be a given data for each given entropic potential.

We write for the current density, the matrix $\operatorname{div}_{\alpha} \operatorname{div}_{\beta} j_{\alpha \beta}$, where $\operatorname{div}_{\alpha}$ is the divergence taken over the energy surface $\alpha$, and $\alpha$ is also viewed as a thermodynamical length scale temperature represented by a thermodynamical functions ratio. $\operatorname{div}_{\alpha}$ is an operator acting on $\alpha$ component of $j_{\alpha \beta}$.

According to the general thermodynamical law [7, 8], we have: $\frac{d \ln T}{d \tau}=-\frac{\left(\frac{\partial V}{\partial \tau}\right)_{P}}{\left(\frac{\partial Q}{\partial P}\right)_{\tau}}$.

Concerning the entropies thermodynamical length scales, A is a potential distribution viewed as a transformation class, which keeping invariant the entropic potential operator, the second Landau condition curlA $=\mathrm{B}$ is replaced by $\overline{A_{\alpha \beta}}=\left(\operatorname{curl}_{\alpha} \operatorname{curl}_{\beta} A_{\alpha} A_{\beta}\right)^{1 / 2}$ in such a way [9-11]

$$
\begin{aligned}
& \frac{\partial^{2} \mathrm{~A}_{\alpha \beta}}{\partial \mathrm{E}_{\alpha} \partial \mathrm{E}_{\beta}}=\overline{\mathrm{S}}_{\alpha \beta}=-5.0396 \cdot 10^{-15}\left(\ln _{\beta} \Delta \Gamma\right)= \\
& =\int \frac{1}{\mathrm{~T}} \mathrm{dE}-5.0396 \cdot 10^{-15}
\end{aligned} .
$$

$-5.0396 \cdot 10^{-15}$ is the computation origin of reduced entropy, it doesn't affect the entropic potential (with rank classification as $\int \frac{1}{\mathrm{~T}} \mathrm{dE}-5.0396 \cdot 10^{-15}$ first rank, $-5.0396 \cdot 10^{-15}\left(\ln _{\beta} \Delta \Gamma\right)$ second rank and $\frac{\partial^{2} A_{\alpha \beta}}{\partial E_{\alpha} \partial E_{\beta}}$ third rank).

An entropic potential variation is equivalent to the sum:

$d j_{\alpha \beta}+\hat{T} d S=d A_{\alpha \beta}$.

By analogous way with the fundamental thermodynamical law: $d E=d R+d Q$, this writing is very simplified, because it admits an operatorial transcription, the precedent mentioned writing is correct to describe regions governed by fluctuating order parameters just near the threshold neighbourhood in order of
$-5.0396 \cdot 10^{-15}$ ( $\hat{T} d S_{\alpha \beta}$ may represent an “eigenvalue” of the exchange heat).

According the analogous thermodynamical length scales defined by Landau, we have:

$\frac{d\left(k \ln _{\alpha}\left(k \ln _{\beta} \Delta \Gamma\right)\right)}{d\left(k \ln _{\beta} \Delta \Gamma\right)}=-\frac{\Delta F}{\hat{T} d S_{\alpha \beta}}$.

$\Delta \mathrm{F}$ is representing the free energy density variation of states, which replaces the volume variation in usual thermodynamics.

According to the following calculations:

$d\left(k \ln _{\alpha}\left(k \ln _{\beta} \Delta \Gamma\right)\right)=k^{2} \frac{\mathrm{d}\left(\ln _{\beta} \Delta \Gamma\right)}{\mathrm{k} \ln _{\beta} \Delta \Gamma}=$

$=k \frac{d\left(\ln _{\beta} \Delta \Gamma\right)}{\ln _{\beta} \Delta \Gamma}=\frac{\frac{k}{\Delta \Gamma}}{\ln _{\beta} \Delta \Gamma}=\frac{k}{\Delta \Gamma} \frac{1}{\ln _{\beta} \Delta \Gamma}$

$d\left(\ln _{\beta} \Delta \Gamma\right)=k \frac{1}{\Delta \Gamma}$,

where $\Delta \Gamma$ is the $\beta$ energy of number states, then

$\frac{d\left(k \ln _{\alpha}\left(k \ln _{\beta} \Delta \Gamma\right)\right)}{d\left(k \ln _{\beta} \Delta \Gamma\right)}=\frac{\frac{k}{\Delta \gamma} \frac{1}{\ln _{\beta} \Delta \Gamma}}{\frac{k}{\Delta \Gamma}}=\frac{1}{\ln _{\beta} \Delta \Gamma}$,

$\frac{1}{\ln _{\beta} \Delta \Gamma}=-\frac{\Delta F}{\hat{T} d S_{\alpha \beta}}$, then

$\frac{\hat{T} d S_{\alpha \beta}}{\ln _{\beta} \Delta \Gamma}=-\Delta F$.

The length scale defined as $d S_{\alpha \beta}=-\frac{\ln _{\beta} \Delta \Gamma}{\hat{T}} \Delta F$ expresses that a finite interval $\Delta \mathrm{F}$ is reduced to an infinitesimal $d S_{\alpha \beta}$ by $\ln _{\beta} \Delta \Gamma$ for a given value of $\hat{T}$.

The equivalence between thermodynamical length scales of entropies, and the extremums of

$\int_{\tau_{1}}^{\tau_{2}} \xi(T, \hat{T}, \tau) d \tau$

It will mean that a coherence length scale is resulting as a reduced entropy variation; the multitude of minimums of the above integral will justify the matrix nature of reduced entropy.

Let us we write the entropic action as $s=\int_{\tau_{1}}^{\tau_{2}} \xi(T, \hat{T}, \tau) d \tau$, the reduced entropy will be maximal when it takes the form $\mathrm{S}_{\alpha \beta}=\mathrm{a}_{\alpha \beta} \mathrm{e}^{-i 7.63 \cdot 10^{-12} \mathrm{~s} \alpha \mathrm{s} \beta}$, which means that if this 
form is taken, the whole regions described by the fluctuating order parameters obey the entropic potential $\mathrm{A}_{\alpha \beta}$.

Because of the relation $\hat{e}^{S_{0}} \Delta F=S_{n 0} j_{\alpha \beta}$, we can write for the asymptotic form $\mathrm{S}_{\alpha \beta}=\mathrm{a}_{\alpha \beta} \mathrm{e}^{-\mathrm{i} 7.63 \cdot 10^{-12} \mathrm{~s} \alpha \mathrm{s} \beta}$; the expression:

$$
\begin{aligned}
& \mathrm{a}_{\alpha \beta}\left(-\left(\hbar^{2} \nabla_{\alpha} \nabla_{\beta}\right)^{1 / 2}+\frac{\mathrm{e}^{*}}{\mathrm{c}} \mathrm{A}\right) \times \\
& \times\left(\Psi_{\alpha} \Psi_{\beta}^{*}\right)^{1 / 2} \mathrm{e}^{-\mathrm{i} 7.63828 \cdot 10^{-12} \mathrm{~s} \alpha^{\mathrm{s}} \beta}= \\
& =-A \int\left|\operatorname{div}_{\alpha} \operatorname{div}_{\alpha} j_{\alpha \beta}\right|^{\frac{1}{2}} d t+S_{n 0} j_{\alpha \beta},
\end{aligned}
$$

which gives the "resonant" $j_{\alpha \beta}$. Such $j_{\alpha \beta}$ are induced by the matrix elements of the reduced entropy, for which we have:

$$
\begin{aligned}
& \left|a_{\alpha \beta}\right|^{2}=1 \text { and } \\
& \left\{\begin{array}{l}
S_{\alpha}=\int_{\tau_{1}}^{\tau_{2}} \xi_{\alpha}(T, \hat{T}, \tau) d \tau \\
S_{\beta}=\int_{\tau_{1}}^{\tau_{2}} \xi_{\beta}(T, \hat{T}, \tau) d \tau
\end{array}\right.
\end{aligned}
$$

which are linked by

$$
\begin{aligned}
& \mathrm{c}_{\alpha} \int_{\tau_{1}}^{\tau_{2}} \xi_{\alpha}(\mathrm{T}, \dot{\mathrm{T}}, \tau) \mathrm{d} \tau+\mathrm{c}_{\beta} \int_{\tau_{1}}^{\tau_{2}} \xi_{\beta}\left(\mathrm{T}^{\prime}, \dot{\mathrm{T}}^{\prime}, \tau\right) \mathrm{d} \tau= \\
& \int_{\tau_{1}}^{\tau_{2}} \mathrm{c}_{\alpha} \xi_{\alpha}(\mathrm{T}, \dot{\mathrm{T}}, \tau) \mathrm{d} \tau+\int_{\tau_{1}}^{\tau_{2}} \mathrm{c}_{\beta} \xi_{\beta}\left(\mathrm{T}^{\prime}, \dot{\mathrm{T}}^{\prime}, \tau\right) \mathrm{d} \tau= \\
& =\int_{\tau_{1}}^{\tau_{2}}\left(\mathrm{c}_{\alpha} \xi_{\alpha}+\mathrm{c}_{\beta} \xi_{\beta}\right) \mathrm{d} \tau \sim-5.0396 \cdot 10^{-15}\left(\ln _{\beta} \Delta \Gamma\right) .
\end{aligned}
$$

Physically those $j_{\alpha \beta}$ are eigen results of first order entropies, having the same phases.

For $\mathrm{S}_{\alpha \beta}=\mathrm{a}_{\alpha \beta} \mathrm{e}^{-\mathrm{i} 7.63 \cdot 10^{-12} \mathrm{~s} \alpha \mathrm{s} \beta}$, the equation will be written as:

$$
\left\{\begin{array}{l}
\hat{e}^{S_{0}} \Delta F=S_{\alpha \beta} j_{\alpha \beta}=a_{\alpha \beta} e^{-i 7,63 \cdot 10^{-12} s_{\alpha} s} \\
\hat{e}^{S_{0}} \Delta F=a_{\alpha \beta} e^{-i 7,63 \cdot 10^{-12} s_{\alpha} \beta}
\end{array}\right.
$$

The expression (10a, b) is giving the "suffocating law" of first order entropies, this is caused by the fact that, the first order entropy converging to the nullity of ancient pattern, will forge an entropy of second order.
$\hat{e}^{S_{0}}$ is a particular operator playing the role of "effective attractor", by consummating the statistical weights of first order belonging to the wave functions, will forge a state with entropy of second order.

\section{Matrix description context for the stabilized superconducting state}

In this work, we consider the "Landau states causal heredity”, this term was used by F. Fer in 1956. The objectivity or the conservation of causality between probabilities density, is of the first order when the measurement processes is defined as the variation of the classical being action (this action is taken according to the uniformity of time, homogeneity and isotropy of space, and also according to the combination of those fundamental features). Nevertheless, when we consider the measurement process as follows:

a) we consider the entropy as the logarithm of the number of extremals of action integral;

b) we consider only the recursive extremals (corresponding to the configurations heretating linear dimensions each from other, it heretating this by a length scales temperatures interactions);

c) by considering the mean quadratic fluctuation over the set of standard configurations, as in the order of the mean intensity of interaction between recursive configurations, for realising this, a threshold is required when it still $-5.0396 \cdot 10^{-15}$ possibilities for the system to increase its entropy.

d) the threshold of the transition of the Landau coherence length is also estimated, it will be a value with the components:

$\left\{\begin{array}{l}\Delta \xi_{i}(T) \Delta \xi_{j}(T) \\ \nabla \xi_{i}(T) \nabla \xi_{j}(T) \\ \left|\begin{array}{l}\Delta \xi_{i}(T) \Delta \xi_{j}(T) \\ \nabla \xi_{i}(T) \nabla \xi_{j}(T)\end{array}\right|\end{array}\right.$

Our approach follows at the first level (the procedure of establishing the canonical equation as in the classical mechanics, the temperature lead to superconductivity, but not guarantee its stability independently to the temperature itself, this means that the macroscopicity of systems in nature will be considered as the shadow of physical conditions, we can reach a superconducting state by decreasing the number of configurations corresponding to a system with action variation is less than the Boltzmann constant, in such a way, the system will be have as to be independent to the temperature.

The entropic potential $\mathrm{A}_{\alpha \beta}$ is in the ancient description appearing latent or absent according to the mean of Landau integral. 


\section{Asymptotic expression for the entropic potential}

The entropic potential must follow the law

$$
A_{\alpha \beta}=c_{\alpha} c_{\beta} \mathrm{e}^{-i 7.63 \cdot 10^{-12} s_{\alpha} s} \text {. }
$$

It is a bi-complex potential, with the modulus is as a measurement of the recursivity of two sets of configurations $\left(\left|c_{\alpha} c_{\beta}\right|^{2}\right.$ must be universal, its constancy implies $\left.\overline{A_{\alpha \beta}}=\left(\operatorname{curl}_{\alpha} \operatorname{curl}_{\beta} A_{\alpha} A_{\beta}\right)^{1 / 2}\right)$. In general one gauge condition is imposed in such a way that $\int\left(\operatorname{curl}_{\alpha} \operatorname{curl}_{\beta} A_{\alpha} A_{\beta}\right)^{1 / 2} d t$ must have extremals (the littlest are chosen).

Here, the interpretation of entropic potential will take the mean of a choice faculty, on one hand, brought on the birth of entropy deflection of the set of standard configurations to the mean entropy of the same set under the "cold"; on another hand, of selection rules of wave functions candidate to generate recursive conditions.

This will be not assumed as a contradiction with GL equations and with the natural character of values, because the entropic potential before the appearance of superconductivity existed as an amount of first order or a function of value of first order.

Those physical considerations do not intend a kind of amendment, but the natural passage to a description, which include the condition of stability of the superconducting state, using the $\mathrm{A}_{\alpha \beta}$ potential.

The concept of heredity introduced will replace the causality limit (the causality term implies that a state is "extracted" from another, all extracted and absolutely, but heredity implies that a state will be extracted from another, not all extracted and not absolutely).

In our case, the action of a potential distribution on a wave function, will generate "a vacuum which must be paved by a set of configurations, which number is as a great, as it still not possibilities for the system to display over, less than $-5.0396 \cdot 10^{-15}$,.

\section{Gauge transition definition}

1. Against which a potential distribution must submit a wave function to obtain an order parameter propagating with Landau length coherence (it will be a gauge imposed to the vector potential in regard to wave functions).

2. Against which conditions must be submit the whole of set of configurations above, to obtain a fluctuation replacing the order parameter and propagating according to trio $\left(\Delta \xi_{i} \Delta \xi_{j}, \nabla \xi_{i} \nabla \xi_{j}, \begin{array}{cc}\Delta \xi_{i} & \nabla \xi_{j} \\ \nabla \xi_{i} & \Delta \xi_{j}\end{array}\right)$.

This will be a gauge of second order with regard to the ordinary wave function. The interference of two wave functions described as a parameterised pavement is limited by the quantum and thermodynamical requirements represented by the two constants: $7.63 \cdot 10^{-12}$ and $2.76 \cdot 10^{-6}$.

We shall say that the proposition $\Psi \Psi^{*}$ is $|\Psi|^{2}$ and replaced by the propositions that $\Delta \Psi \Delta \Psi^{*} \sim c_{1}=$ $7.63 \cdot 10^{-12}, \quad \nabla \Psi \nabla \Psi^{*} \sim c_{2}=2.76 \cdot 10^{-6}$, and $\left|\begin{array}{l}\Delta \Psi \Delta \Psi^{*} \Delta \Psi^{*} \Delta \Psi \\ \nabla \Psi \nabla \Psi^{*} \nabla \Psi^{*} \nabla \Psi\end{array}\right| \times c_{3}$ are three uncertainty principles.

First expression means the limitations imposed to the pavement with regard to the linear dimensions.

Second one means the limitations imposed to the pavement with regard to the orientations.

Third one means the limitations imposed to the pavement with regard to the birth of mixed current density, which will be corresponding to the transition of magnetic features.

The limitations imposed to the pavement, will be considered as the origin of the deflexion of entropy, and will give a great number of minimums for the Landau functional. The Landau phenomenological parameters are the results of measurement process of first order, $\mathrm{A}_{\alpha \beta}$ will be a result of "the transition of measurement process", $\mathrm{A}_{\alpha \beta}$ is governing the order of fluctuations of the set of the configurations above, which will appear as a result of measurement process here "application of entropic potential”.

This description permit the appearance of relations between entropic potential and fluctuations described above, as erecursively, where every equilibrium state between fluctuations and entropic potential, will be recursive partial equilibrium state (the measurement process generate results, results will be dictated how to be generated, it is a kind of the J.A. Wheeler law).

According to these considerations, the mean of value of the entropic potential must follow the law:

$\overline{A_{\alpha \beta}} \times c \frac{\Delta \Gamma \Delta \Gamma^{\prime}}{\xi^{2}(T)}$.

This general expression will follow the limitations imposed by the pavement described above as follows:

$$
\begin{aligned}
& \overline{A_{\alpha \beta}} \approx c_{1} \frac{\Delta \Gamma_{\alpha} \Delta \Gamma_{\beta}}{\Delta \xi_{\alpha}(T) \Delta \xi_{\beta}(T)}, \\
& \underline{A_{\alpha \beta}} \approx c_{2} \frac{\nabla \Gamma_{\alpha} \nabla \Gamma_{\beta}}{\nabla \xi_{\alpha}(T) \nabla \xi_{\beta}(T)}, \\
& \overline{A_{\alpha \beta}} \approx\left|\begin{array}{ll}
c_{11} & c_{12} \\
c_{21} & c_{22}
\end{array}\right| \frac{\left|\begin{array}{ll}
\Delta \Gamma_{\alpha} & \nabla \Gamma_{\beta} \\
\nabla \Gamma_{\alpha} & \Delta \Gamma_{\beta}
\end{array}\right|}{\left|\begin{array}{ll}
\Delta \xi_{\alpha}(T) & \Delta \xi_{\beta}(T) \\
\nabla \xi_{\beta}(T) & \Delta \xi_{\beta}(T)
\end{array}\right|} .
\end{aligned}
$$

The operator transcription of the variational principle [9], $\iint \Psi(q) \Psi^{*}(q) \varphi\left(q, q^{\prime}\right) d q d q^{\prime}, \quad$ is as follows: 
$\hat{e}^{S_{0}} \Delta=\iint S_{\alpha}(E) S_{\beta}(E) A_{\alpha \beta} d t^{2}$.

$d t^{2}$ is an infinitesimal of second order which generates the transition of the universal undetermination order to an eigen undetermination orders.

The following calculation shows that:

$$
\iint S_{\alpha}(E) S_{\beta}(E) A_{\alpha \beta} d t^{2}=S_{n_{0}} j_{\alpha \beta}
$$

and $\hat{e}^{s_{0}} \Delta F=a_{\alpha \beta} e^{-i 7.63 \cdot 10^{-12} s_{\alpha} s \beta} j_{\alpha \beta}$, then

$$
\iint S_{\alpha}(E) S_{\beta}(E) A_{\alpha \beta} d t^{2}=a_{\alpha \beta} e^{-i 7.63 \cdot 10^{-12} s_{\alpha} s} j_{\alpha \beta},
$$

$\frac{d^{2}}{d t^{2}}\left(\hat{e}^{s_{0}} \Delta F\right)=S_{\alpha}(E) S_{\alpha}(E) A_{\alpha \beta}+\left|c^{2}\right|$,

$\frac{\mathrm{d}}{\mathrm{dt}}\left(\frac{\mathrm{i}}{\hbar}\left[\mathrm{He}^{\mathrm{S}_{0}}-\mathrm{e}^{\mathrm{S}_{0} \mathrm{H}}\right]\right) \Delta \mathrm{F}=\mathrm{S}_{\alpha}(\mathrm{E}) \mathrm{S}_{\beta}(\mathrm{E}) \mathrm{A}_{\alpha \beta}+||^{2}$.

$|c|^{2}$ ensures the recursivity between the fluctuations (by fluctuation we mean the one of system fluctuating over a number of configurations) and the entropic potential, this leads to the uniformity of $A_{\alpha \beta}$, it does not change sign (the reverse means the appearance of extremal symmetries or a dislocated configurations).

With regard to the energies, the expression of the mean value of entropic potential is:

$$
\begin{aligned}
& \overline{\mathrm{S}}_{\alpha \beta}=\frac{\partial^{2} \mathrm{~A}_{\alpha \beta}}{\partial \mathrm{E}_{\alpha} \partial \mathrm{E}_{\beta}}=-5.0396 \cdot 10^{-15}\left(\ln _{\beta} \Delta \Gamma\right)= \\
& \int \frac{1}{\mathrm{~T}} \mathrm{dE}-5.0396 \cdot 10^{-15} .
\end{aligned}
$$

This fact shows that $\frac{1}{\left(\mathrm{~T}-\mathrm{T}_{\mathrm{c}}\right)} \mathrm{dE}-5.0396 \cdot 10^{-15}$ implies a computation origin of reduced entropy, situated not as far as it exist a real situation, where the integral is less than $-5.0396 \cdot 10^{-15}$. The appearance of entropic potential is per pavement, and the reduced entropy is per ratios of displaying faculties.

Entropic potential is existing as a latent form at the first order , it seems as a Landau potential distribution, or different by a constant potential (the Landau distribution is in this case a tangent space to the flat manifold represented by entropic potential), by the transition it will transit in a value of second order with regard to the wave function, the entropic potential is collected in terms of effective action variation density per pavement as: $\frac{\delta^{3} \mathrm{~s}}{\delta \mathrm{q}_{\mathrm{i}} \delta \mathrm{q}_{\mathrm{j}} \delta \mathrm{q}_{\mathrm{k}}}, \quad \frac{\delta^{3} \mathrm{~s}}{\delta \mathrm{q}_{\mathrm{i}} \delta \mathrm{q}_{\mathrm{j}} \delta \mathrm{q}_{\mathrm{k}}}$, $\frac{\delta^{3} \mathrm{~s}}{\delta \varphi_{\mathrm{i}} \delta \varphi_{\mathrm{j}} \delta \varphi_{\mathrm{k}}}$, and their linear or non linear

\section{Conclusion}

The equivalence $\frac{\partial^{2} \mathrm{~A}_{\alpha \beta}}{\partial \mathrm{E}_{\alpha} \partial \mathrm{E}_{\beta}} \sim \int \frac{1}{\mathrm{~T}} \mathrm{dE}-5.0396 \cdot 10^{-15}$ leads to estimate the contraction of macroscopic levels as in the order of $d E=8.75 \cdot 10^{-13} \mathrm{erg}$, a part of the interval $\Delta \mathrm{E}$ (in the order of mean fluctuation of macroscopic level), will seem disappearing in the meaning of standard entropy measured under the Heisenberg undetermination, but really this part doesn't disappear, but transformed to correspond to recursive configurations. On another hand, this amount of energy $\mathrm{dE}$ represents the displaying of $\Delta \mathrm{E}$ over inner freedom degrees of the system, which corresponds to the inner dimensions of the antisymmetric order parameter, one inner local dimension is reversed to be a global external dimensions.

Such considerations are making that the interaction processes will be viewed as excluded inner dimensions to realise external.

\section{References}

1. A. Ardelea, G.F. Carey, A. Pardhanani, and W. Richardson, Simulation of macroscopic superconductivity for microelectronics // Physica C, 341-348, p. 2649-2650 (2000).

2. A. Aftalio, E. S\&ndier, S. Serfaty: Pinning phenomena in the Ginzburg Landau model of superconductivity // J. Math. Pures Appl. 80(3), p. 339-372 (2001).

3. J. Deang, Q. Diu and M. Gunzburger, Modeling and computation of random thermal fluctuations and material defects in the Ginzburg-Landau model for superconductivity // J. Comput. Phys. 181, p. 45-67 (2002).

4. C. Berthod and B. Giovannini, Cooperon propagator description of high temperature superconductivity // Physica C 364-365, p. 467-470 (2001).

5. X. Hu, Bicritical phenomena and scaling of $\mathrm{O}(5)$ model // Physica A 321, p. 71-80 (2003).

6. W.J Kossler, C. Fetsch and K. Baranowski, Magnetic field distributions from longitudinally disordered pancake vortices // Physica B 326, p. 300-304 (2003).

7. L. Landau, and E. Lifschitz, Statistical Physics. MIR Edition, Moscow,1969 (in Russian).

8. L. Landau, and E. Lifschitz, Quantum Mechanics. MIR Edition, Moscow, 1969 (in Russian).

9. N. Bohr, Physique Atomiqu et Connaissance Humaine. Editions Gonthier, by Gauthier Villars, Paris, 1961.

10. M. Thinkham, Introduction to superconductivity. Second Edition, McGraw Hill Inc, 1996.

11. Charles W. Misner, Kip S. Thorne, and J.A. Wheeler, Inspired from vacuum fluctuations. "Gravitation" Company, San Fransisco, 1973. 